\title{
Áreas protegidas prioritarias para la investigación y el monitoreo de la biodiversidad en Nicaragua
}

\author{
Antonio Mijail Pérez ${ }^{(1)}$, Marlon Sotelo ${ }^{(1,2)}$, Isabel Siria ${ }^{(2,1)}$ y Adolfo López SJ (1) \\ 1. Centro de Malacología y Diversidad Animal, Universidad Centroamericana, Apdo.69, Managua, Nicaragua. E-mail: ampp@ns.uca. \\ edu.ni; \\ 2. Asociación Gaia.
}

Recibido: septiembre de 2006 / Aceptado: octubre de 2006

LA PRIORIZACIÓN DE ÁREAS PROTEGIDAS PARA LA INVESTIGACIÓN Y EL MONITOREO de la biodiversidad es una necesidad de la mayoría de los países, incluyendo los más desarrollados, ya que la disponibilidad de recursos financieros para estos efectos es siempre limitada. En el presente trabajo se presentan las áreas protegidas seleccionadas como prioritarias para la realización de monitoreo e investigación en biodiversidad en Nicaragua, así como se detalla la herramienta multicriterio diseñada para tal efecto. Aplicando la citada herramienta se obtuvo un listado de 16 áreas el cual fue completado por consulta con la comunidad científica, por dos áreas más para un total de 18 áreas protegidas prioritarias para el monitoreo y la investigación de la biodiversidad en el país.

Palabras clave: Reservas forestales-Nicaragua / diversidad biológica / parques naturales

\section{Introducción}

La necesidad e importancia de establecer programas y sistemas de monitoreo de la biodiversidad ha sido señaladas por diversos autores desde hace años (McGuinnes, 1994; Bridgewater, 1999; McNeely, 2002), y es de consenso total que es imposible incidir en los procesos de toma de decisiones si no existen estos programas y no se cuenta por consiguiente con información fiable y sistemática.

Según Rallo (1998) la sistematización del conocimiento es la base para que el mismo se considere científico y, por consiguiente, pueda ser utilizado por la sociedad y por los tomadores de decisión. Es por ello que la sistematización de la información sobre biodiversidad es una de las bases para el desarrollo del país en este ámbito.

En este sentido es importante considerar que incluso en países del llamado "primer 
mundo", es necesario realizar algún tipo de priorización para la investigación y el monitoreo. Con mucha más razón en países como Nicaragua con serias limitaciones económicas. Una iniciativa muy importante fue la metodología de la efectividad de manejo de las áreas protegidas presentada por PROARCA/CAPAS, basada en el método desarrollado por The Nature Conservancy (TNC), la que enfatiza aquellos aspectos relacionados con el manejo de las áreas.

En Nicaragua, un antecedente de gran importancia fue el aportado en el marco del Programa de Investigación y Monitoreo en Áreas Protegidas de Nicaragua (vid. Pérez et al. 2005), en el cual se propone una herramienta para la valoración holística de las áreas protegidas, la cual, a diferencia de aquella propuesta por TNC, tiene un enfoque mayormente estructural que hace énfasis en las características de las áreas más que en su manejo.

En este artículo se presentan las áreas seleccionadas como prioritarias para el desarrollo de un Programa de investigación y monitoreo de la biodiversidad en Nicaragua, y se detalla la herramienta que se propuso para tal efecto.

\section{Contexto}

Según Mittermeier et al (2000), América Central, y por consiguiente Nicaragua, está considerada como uno de los 25 "hot spots" citados en el nivel mundial (Ilustraciones 1 y 2). Los hotspots se establecen simultaneando criterios de amenaza y endemismo. Teniendo en cuenta lo anterior, toda Nicaragua, y especialmente sus áreas protegidas, son prioridad de cara a la conservación.

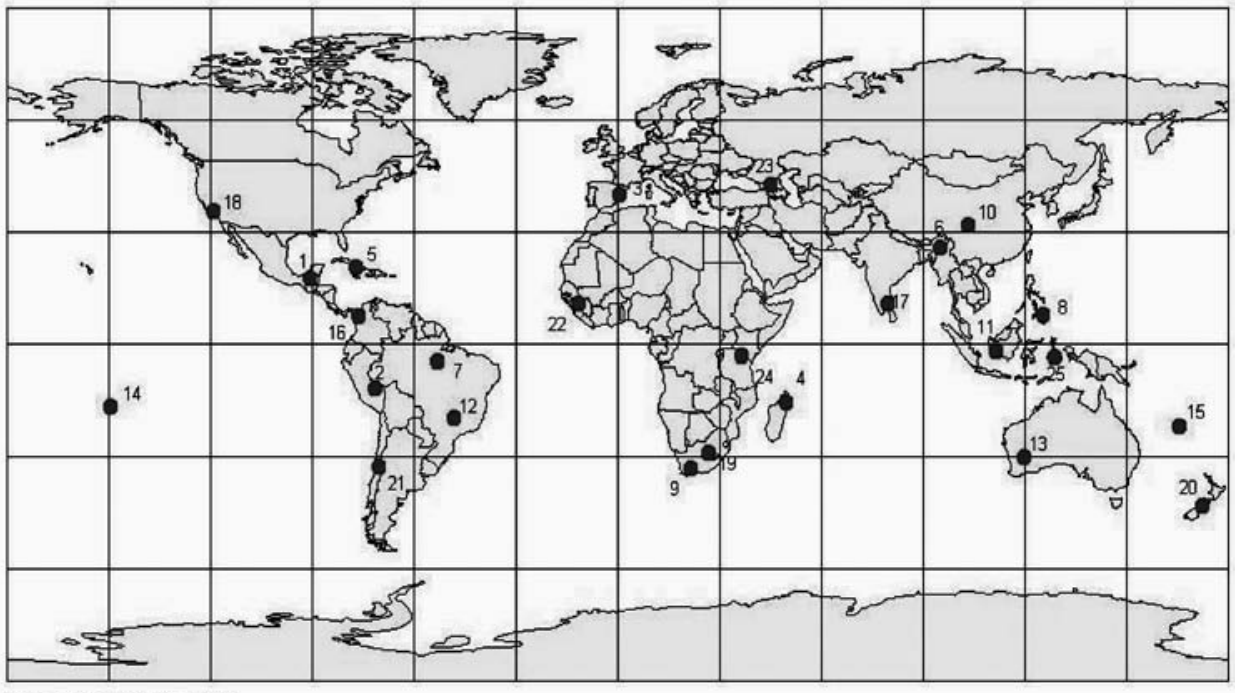

Fuente: Mitternayer, et al. 2000

Ilustración 1. Puntos calientes de la diversidad global (Según Mittermeier et al., 2000). 


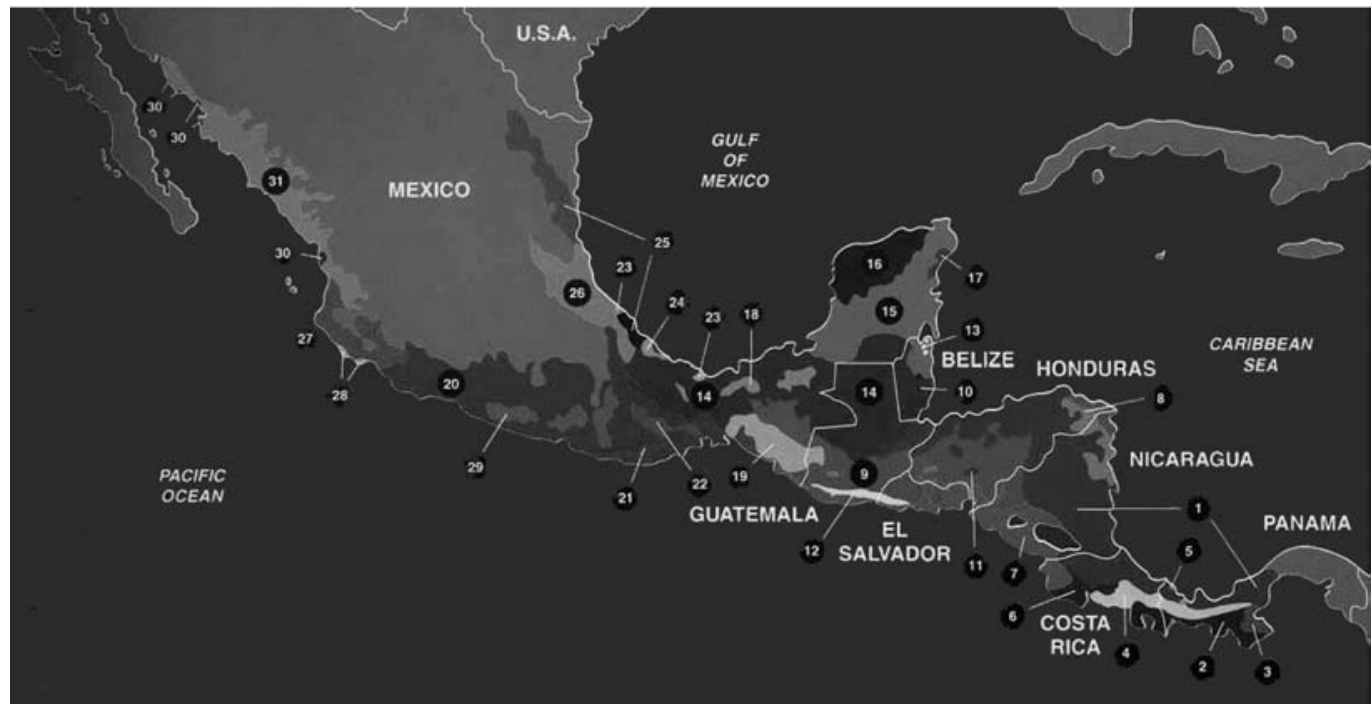

Ilustración 2. El hotspot de América Central.

Los datos de diversidad de especies son altos en los casos que se conocen, pero es notable, en el caso de los invertebrados, la ausencia de información (Cuadros 1 y 2).

Cuadro 1. Datos comparativos de diversidad global y nacional. Vertebrados y plantas.

\begin{tabular}{|c|c|c|c|c|c|c|}
\hline País & Mamíferos & Aves & Reptiles & Anfibios & Peces & $\begin{array}{c}\text { Plantas } \\
\text { Superiores }\end{array}$ \\
\hline Nicaragua & 251 & 676 & 172 & 62 & 643 & 6500 \\
\hline El Mundo & 4327 & 9672 & 6550 & 4000 & 22000 & 240000 \\
\hline$\%$ & 5.80 & 6.98 & 2.62 & 1.55 & 2.92 & 2.71 \\
\hline
\end{tabular}

Cuadro 2. Datos comparativos de diversidad global y nacional. Invertebrados.

\begin{tabular}{|c|c|c|c|c|c|}
\hline País & Corales & Moluscos & Insectos & Crustáceos & R otífer os \\
\hline Nicaragua & 58 & 2000 & 8514 & 88 & 57 \\
\hline El Mundo & Dnd.* & 60000 & 1,200000 & 4000 & Dnd.* \\
\hline$\%$ & -- & 3.33 & 0.71 & 2.2 & -- \\
\hline
\end{tabular}

* Dato no disponible

Estos valores de diversidad son realmente impresionantes si se tiene en cuenta el área del país (Ilustración 3). 


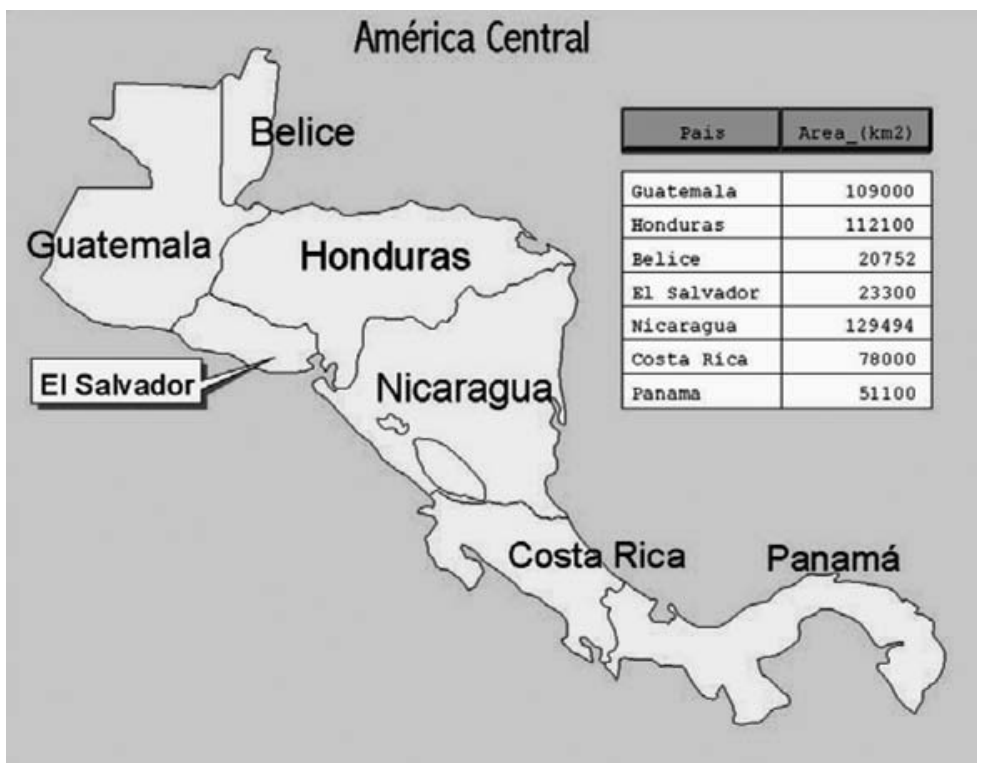

Ilustración 3. Superficie de los países de la región.

Por consiguiente, al margen de los criterios utilizados para la selección de nuestras áreas protegidas, las mismas deben contener un importante porcentaje de la biodiversidad nacional, y actualmente las áreas protegidas representan el 17\% del territorio nacional (Ilustración 4), lo que constituye un área relativamente significativa del mismo.

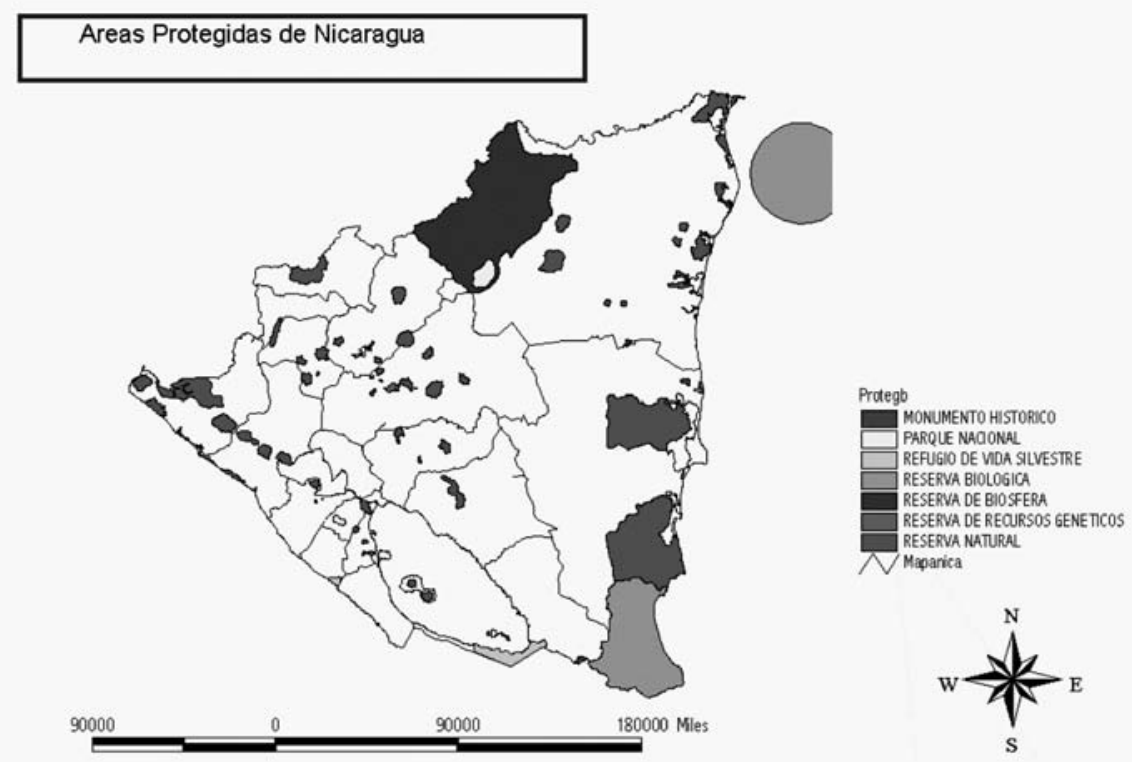

Ilustración 4. Mapa de las áreas protegidas de Nicaragua. 
A los efectos de la Convención de Diversidad Biológica (CDB) (BOE, 1994), por ÁREA PROTEGIDA se entiende "un área definida geográficamente que haya sido designada o regulada y administrada a fin de alcanzar objetivos específicos de conservación”.

Según la UICN (1994), un ÁREA PROTEGIDA se entiende como "un área de tierra y/o de mar dedicada especialmente a la protección y mantenimiento de la diversidad biológica, y de recursos naturales y culturales asociados, manejados mediante medios legales u otros que sean efectivos"

De acuerdo con el REGLAMENTO DE AREAS PROTEGIDAS DE NICARAGUA y la LEY 217 (Ley General del Medio Ambiente), son Áreas Protegidas "las que tienen por objeto la conservación, el manejo racional y la restauración de la flora, fauna silvestre y otras formas de vida, así como la biodiversidad y la biosfera, se pretende con ello restaurar y conservar fenómenos geomorfológicos, sitios de importancia histórica, arqueológica, cultural, escénicos o recreativos".

Las categorías de manejo nacionales y de la UICN se presentan en el Cuadro 3. Se incluye también un análisis que permite destacar las diferencias existentes entre estas categorías de cara a su mejor y más correcta aplicación.

Cuadro 3. Categorías de manejo de Áreas Protegidas en Nicaragua.

\begin{tabular}{|c|c|c|c|c|}
\hline $\begin{array}{l}\text { Categoría en } \\
\text { Nicaragua }\end{array}$ & Categoría UICN & Elemento diferencial & $\begin{array}{l}\text { Cantidad } \\
\text { de áreas }\end{array}$ & $\begin{array}{l}\text { Permite } \\
\text { intervención }\end{array}$ \\
\hline Reserva biológica & $\begin{array}{l}\text { Reserva Natural } \\
\text { estricta }\end{array}$ & $\begin{array}{l}\text { Áreas que poseen ecorregiones } \\
\text { y/o especies representativas. }\end{array}$ & 2 & NO \\
\hline Parque Nacional & Parque Nacional & $\begin{array}{l}\text { Área idónea para proteger uno o } \\
\text { más ecosistemas y hábitats así } \\
\text { como rasgos de interés cultural. }\end{array}$ & 3 & NO \\
\hline $\begin{array}{l}\text { Refugio de Vida } \\
\text { Silvestre }\end{array}$ & $\begin{array}{l}\text { Área de manejo de } \\
\text { hábitats/especies }\end{array}$ & $\begin{array}{l}\text { Área sujeta a intervención activa } \\
\text { para garantizar el mantenimiento } \\
\text { de los hábitats y/o para satisfacer } \\
\text { las n ecesidades de determinadas } \\
\text { especies o comunidades } \\
\text { animales. }\end{array}$ & 4 & SI \\
\hline Reserva Natural & $\begin{array}{l}\text { Área de manejo de } \\
\text { hábitats/especies }\end{array}$ & $\begin{array}{l}\text { Área con especies de interés que } \\
\text { genere beneficios ambientales de } \\
\text { interés nacional o regional. }\end{array}$ & 60 & SI \\
\hline $\begin{array}{l}\text { Reserva de recursos } \\
\text { genéticos }\end{array}$ & $\begin{array}{l}\text { Área de manejo de } \\
\text { hábitats/especies }\end{array}$ & $\begin{array}{l}\text { Área que protege algunas } \\
\text { especies por el interés de sus } \\
\text { recursos genéticos, los cuales } \\
\text { pueden ser utilizados para } \\
\text { mejoramiento genético de } \\
\text { especies de interés económico o } \\
\text { alimenticio. }\end{array}$ & 2 & \begin{tabular}{c}
\multicolumn{1}{c}{ SOLO } \\
PARA \\
FINES DE \\
MANEJO
\end{tabular} \\
\hline Monumento nacional & Monumento natural & $\begin{array}{l}\text { Área que contiene rasgos } \\
\text { naturales y/o histórico -culturales } \\
\text { de valor destacado o excepcional } \\
\text { por su rareza implícita, sus } \\
\text { calidades representativas o } \\
\text { estéticas. }\end{array}$ & 2 & SI \\
\hline
\end{tabular}




\begin{tabular}{|l|l|l|c|c|}
\hline Monumento histórico & Monumento natural & $\begin{array}{l}\text { Territorios que contienen uno 0 } \\
\text { varios rasgos culturales, } \\
\text { históricos o arqueológicos de } \\
\text { importancia nacional o } \\
\text { internacional asociados a áreas } \\
\text { naturales. }\end{array}$ & 1 & NO \\
\hline $\begin{array}{l}\text { Paisaje terrestre y } \\
\text { marino protegido }\end{array}$ & $\begin{array}{l}\text { Paisaje terrestre y } \\
\text { marino protegido }\end{array}$ & $\begin{array}{l}\text { La interacción entre el hombre y } \\
\text { la naturaleza ha producido una } \\
\text { zona con importantes valores } \\
\text { estéticos, ecológicos y culturales. }\end{array}$ & 1 & SI \\
\hline Reserva de Biosfera & NO TIENE & $\begin{array}{l}\text { Áreas que integran diferentes } \\
\text { Categorías de manejo y } \\
\text { administradas integralmente, } \\
\text { logran un desarrollo sostenible. }\end{array}$ & 2 & SI \\
\hline
\end{tabular}

\section{Metodología}

136 RECOPILACIÓN DE INFORMACIÓN: Se recopiló información en la biblioteca del MARENA, dirección del SINAP, proyectos del MARENA, centros de documentación de ONG, otras bibliotecas y centros de documentación especializados en el país, así como la Internet. Se revisaron las siguientes obras:

\section{Planes de Manejo:}

1. Parque Nacional Volcán Masaya.

2. Parque Nacional Archipiélago de Zapatera.

3. Refugio de Vida Silvestre Río Escalante-Chacocente.

4. Refugio de Vida Silvestre Los Guatuzos.

5. Reserva de Recursos Genéticos Yucul.

6. Reserva Natural Estero Padre Ramos.

7. Refugio de Vida Silvestre Isla Juan Venado.

8. Reserva Natural Cerro Tisey Estanzuela.

9. Reserva Natural Cerro Datanlí El Diablo.

10. Reserva Natural Cerro Arenal.

11. Reserva Natural Cerro Musún.

12. Reserva Natural Cerro Apante.

13. Reserva de Biosfera Bosawás. * Reserva Natural Bosawás.

* Reserva Natural Kilambé.

* Reserva Natural Cerro Cola Blanca (En proceso).

* Parque Nacional Saslaya.

* Reserva Natural Macizo de Peñas Blancas.

* Reserva Natural Cerro Bana Cruz.

14. Refugio de Vida Silvestre Río San Juan.

\section{Planes de manejo en proceso:}

1. Reserva Biológica Indio Maíz.

2. Reserva de Biosfera Cayos Miskitos y Franja Costera Inmediata. 
3. Monumento Histórico Fortaleza La Inmaculada.

4. Refugio de Vida Silvestre La Flor.

5. Reserva Natural volcán Cosigüina.

6. Reserva de Recursos Genéticos Apacunca.

7. Reserva Natural Delta del Estero Real.

8. Reserva Natural Complejo Volcánico San Cristóbal.

9. Reserva Natural Península de Chiltepe.

10. Laguna de Asososca.

11. Reserva Natural Laguna de Tiscapa.

12. Reserva Natural Laguna de Tisma.

13. Reserva Natural Volcán Mombacho.

14. Reserva Natural Laguna de Apoyo.

15. Reserva Natural Cordillera de Dipilto y Jalapa.

16. Reserva Natural Tepesomoto-Pataste.

17. Reserva Natural Cerro Cola Blanca.

18. Refugio de Vida Silvestre Chocoyero El Brujo.

19. Reserva Natural Cerro Wawashan.

20. Reserva Natural Cerro Silva.

\section{Documentos:}

- CCAD (2003). Estado del sistema centroamericano de áreas protegidas. Informe de síntesis regional. Managua: CCAD. (Edición bilingüe).

- MARENA (2003). Estado actual del ambiente en Nicaragua 2003. II Informe Geo. Managua: Impresión comercial La Prensa.

- MARENA (2004). Indicadores ambientales de Nicaragua. Volumen I. Managua: Impresión comercial La Prensa.

- SNU-BID-SECEP-BM-CONPES (2003). Metas de desarrollo. Seguimiento a la cumbre del milenio. Nicaragua. Primer Informe. Managua: Imprimatur.

- $\quad$ ZEAS, M.; QUINTERO, B.; SÁNCHEZ, M.; MOTHA, S. (2002).Áreas protegidas. Informe Nacional. Managua: GEF/PNUD.

Consultas con los actores: En esta fase del trabajo se realizaron entrevistas a informantes claves dentro del MARENA central, a miembros de la comunidad científica, tanto de universidades como independientes, así como algunos ONG.

\section{ANÁLISIS DE LA INFORMACIÓN.}

Criterios de prioridad de las áreas protegidas: Para la selección de las áreas protegidas prioritarias del Estado (MARENA), se establecieron una serie de criterios de diferente tipo. Con los citados criterios se elaboró una matriz multicriterio que fue procesada con el mismo formato de una matriz de impacto ambiental (vid. CONESA, 1995). La estructura de la matriz será como sigue: 


\begin{tabular}{|c|c|c|c|c|c|}
\hline \multirow[t]{2}{*}{ Áreas } & \multicolumn{4}{|c|}{ Criterios } & \multirow{2}{*}{$\begin{array}{c}\text { Puntuación } \\
\text { (IVP) }\end{array}$} \\
\hline & I & II & III & IV... & \\
\hline \multicolumn{6}{|l|}{ Sureste } \\
\hline \multicolumn{6}{|l|}{ Bosawás } \\
\hline \multicolumn{6}{|l|}{ Chacocente } \\
\hline$\ldots$ & & & & & \\
\hline
\end{tabular}

La selección de las áreas fue realizada de acuerdo a la puntuación obtenida mediante el cálculo de un Índice de Valor de la Prioridad (IVP), que es un índice cuantitativo aditivo que estará dado por la expresión:

$$
\mathrm{IVP}=\Sigma \mathrm{CP}=\Sigma \mathrm{AS}+\Sigma \mathrm{AA}+\Sigma \mathrm{AB}+\Sigma \mathrm{AL}+\Sigma \mathrm{CG}+\Sigma \mathrm{PBS}
$$

Donde:

CP: Criterios de Prioridad.

138 AS: Aspectos Sociales.

AA: Aspectos Administrativos.

AB: Aspectos Biológicos.

AL: Aspectos Logísticos.

CG: Contexto Geopolítico.

PBS: Producción de Bienes y Servicios.

Según el análisis multicriterio realizado, aquellas áreas que resulten evaluadas con 25 puntos o más se entenderá que reúnen los criterios necesarios para ser elegidas prioritarias por parte del MARENA. La cantidad de puntos establecida como límite inferior (25 puntos) constituye el percentil 60 sobre el valor de 100 que en este caso es de 42 puntos como máximo. Las áreas evaluadas sólo con criterios biológicos tuvieron un máximo posible de 11 puntos. Las categorías de los criterios de prioridad serán establecidas en cinco tipos de categorías:

1. Presencia/ Ausencia (SI/NO) que será codificado como:

$\mathrm{S}=1$

$\mathrm{N}=0$

2. Tres niveles para las categorías de manejo:

3

2

1

3. Alta/ Media/ Baja (A/M/B) que será codificado como:

$\mathrm{A}=3$

$\mathrm{M}=2$

$\mathrm{B}=1$

4. Alta/ Media/ Baja/Nula (A/M/B/N) que será codificado como:

$\mathrm{A}=3$

$\mathrm{M}=2$

$\mathrm{B}=1$

$\mathrm{N}=0$ 
5. Muy Alta/Alta/ Media/ Baja/Nula (MA/A/M/B/N) que será codificado como:

$\begin{array}{ll}\mathrm{MA}= & 4 \\ \mathrm{~A}= & 3 \\ \mathrm{M}= & 2 \\ \mathrm{~B}= & 1 \\ \mathrm{~N}= & 0\end{array}$

\section{Criterios propuestos:}

\section{Aspectos Sociales (AS).}

A. Grupos humanos (Presencia/ Ausencia).

1

0

Es importante tener en cuenta la dinámica social que se presenta, tanto dentro del área protegida como de su entorno, así como también la participación de los grupos humanos que permita compartir la autoridad y responsabilidad de los recursos que se protegen, de cara a conseguir una administración más eficiente del manejo de sus recursos.

Según Milán (2004), los Sistemas Humanos están bajo un proceso constante de interacción entre los diferentes componentes que lo integran. Como consecuencia de esta interacción se generan presiones que pueden ser internas, o sea, que repercuten dentro de los propios sistemas humanos, o presiones externas, que se reflejan sobre los componentes de los Sistemas Naturales.

Estas presiones actúan como impactos y la prioridad humana debe tener como premisa la mitigación, la preservación o la conservación, ya que los efectos de esas presiones definen la Calidad Ambiental.

Según Milán (2004), las principales formas de presión ambiental debidas a la influencia humana en el espacio se pueden agrupar en las siguientes categorías:

1. Uso del suelo

2. Prácticas administrativas

3. Consumo

B. Comunidades indígenas (Presencia/ Ausencia).

1

0

Determinar la presencia de grupos indígenas dentro de las áreas protegidas con el objetivo de valorar la diversidad cultural del área, que es uno de los intereses del presente Programa.

C. Interés de la cooperación internacional (A, M, B, N).

3 Más de tres agencias o entidades con presencia en el área.

2 Dos o tres agencias o entidades con presencia en el área.

1 Una agencia o entidad con presencia en el área.

0 Ninguna agencia 0 entidad con presencia en el área.

Según García (2002), éste es un criterio de gran importancia a tener en cuenta.

D. Interés de la comunidad científica (A, M, B, N).

3 Más de tres grupos o investigadores han realizado o están realizando proyectos de 
investigación en el área.

2 Dos o tres grupos o investigadores han realizado o están realizando proyectos de investigación en el área.

1 Algún grupo o investigador ha realizado o está realizando proyectos de investigación en el área.

0 No se han realizado o están en curso proyectos de investigación científica en el área.

\section{Aspectos Administrativos (AA).}

A. Categoría de manejo $(3,2,1)$ :

3 Reservas de biosfera.

2 Reserva Biológica, Parque Nacional, Reservas Genéticas

1 Reservas naturales, RVS, MH, MN, PT/MP.

Es la valoración de las características biofísicas y socioeconómicas intrínsecas del área y los objetivos de conservación que puede cumplir. Para esto agruparemos las categorías en dos niveles diferentes, en dependencia del lugar que ocupe en un orden jerárquico previamente establecido. En esta escala, la categoría de mayor importancia son las RESERVAS DE BIOSFERA (vid. Vozmediano, 2003). El resto de las categorías se agrupa en el mismo nivel, ya que cumple objetivos de manejo diferentes que no pueden ser fácilmente jerarquizados.

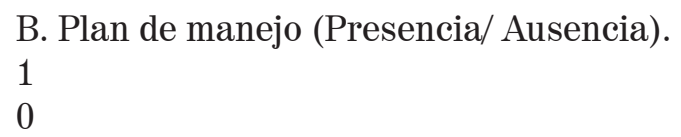

Existencia o no de un plan de manejo como instrumento de gestión que garantice una administración más efectiva en el manejo de los recursos del área protegida.

C. Presencia de Financiamiento (A, M, B).

3 Más de tres agencias o entidades con presencia en el área.

2 Dos o tres agencias o entidades con presencia en el área.

1 Una agencia o entidad con presencia en el área.

0 Ninguna agencia o entidad con presencia en el área.

Ninguna acción que se proponga para la administración de las áreas protegidas puede ser efectiva si no se cuenta con los recursos económicos suficientes, así como con los mecanismos alternos que garanticen el logro de los objetivos propuestos para el desarrollo del área y el cumplimiento de las funciones esenciales inherentes a su categoría de manejo (Mena y Artavia). Para este criterio estableceremos tres niveles de valoración en dependencia del grado de financiamiento con que cuentan las áreas: Alto (A), Medio (M) y Bajo (B).

\section{Aspectos Biológicos (AB).}

A. Diversidad de Ecosistemas (MA, A. M, B, N).

4 Con más de cuatro ecosistemas.

3 Con tres o cuatro ecosistemas.

2 Con dos o tres ecosistemas.

1 Con un ecosistema.

En el taller de técnicos sobre: Conservación de los ecosistemas y la Flora Silvestre in situ y ex situ, como parte de la Estrategia Nacional de Conservación de la Biodiversidad de 
Nicaragua (10.01.01), se concluyó que la única forma de conservar nuestra biodiversidad es in situ, por lo cual es muy relevante e importante desarrollar las Áreas Protegidas (Meyrat, 2001).

B. Presencia de especies emblemáticas (Endemismos, especies bandera, etc.) (MA, A. M, B, N).

4 Con más de cuatro especies.

3 Con tres o cuatro especies.

2 Con dos o tres especies.

1 Con una especie.

0 Ninguna.

C. Presencia de especies invasoras (Presencia/ Ausencia).

1

0

D. Área de descanso de aves migratorias (Presencia/ Ausencia):

1

0

E. Ecosistemas en convenios internacionales (Presencia/ Ausencia).

1

0

Existen ocho áreas protegidas que cuentan con el reconocimiento internacional, de las cuales dos son Reservas de la Biosfera, y son nominadas y reconocidas dentro del programa El Hombre y la Biosfera (MAB) de la UNESCO; las otras seis son reconocidas como sitios de importancia internacional para las aves acuáticas migratorias, según la Convención RAMSAR (MARENA, 2003).

- Reservas de la Biosfera: BOSAWAS y Sureste.

Según Pauquet (En Línea), las Reservas de Biosfera constituyen una categoría de conservación muy adecuada a la realidad latinoamericana, en la medida que busca conciliar el desarrollo y la protección, haciendo compatibles acciones de producción en las zonas de amortiguación y transición, aplicando medidas de protección en las zonas núcleo, y buscando proteger la diversidad biológica y genética del área. Todo esto es concordante con el concepto de desarrollo sostenible, que incluye generar alternativas para las comunidades locales y actores regionales, que son los que definirán la posibilidad efectiva de conservar ecosistemas y especies representativas de la riqueza natural.

- Sitios RAMSAR:

- Refugio de Vida Silvestre Los Guatuzos.

- Reserva Biológica Cayos Miskitos y Franja Costera Inmediata.

- Reserva Natural Delta del Estero Real y Llanos de Apacunca.

- Lago de Apanás-Asturias.

- Refugio de Vida Silvestre Río San Juan.

- Sistema de Humedales de la Bahía de Bluefields. 
- Sistema de Humedales de San Miguelito.

- Reserva Natural Laguna de Tisma.

4. Aspectos Logísticos (AL).

A. Infraestructura básica (Presencia/ Ausencia).

1

0

Existe un total de 12 áreas que cuentan con infraestructura básica y equipo para operar, presencia institucional, presupuesto y personal permanente para la protección, manejo y cierta actividad turística (MARENA, 2001).

B. Equipo idóneo para el manejo del área por cantidad de guarda parques (A, M, B).

3 Botas, capote, machete, mecate, binoculares, guías de campo, GPS y mapas de la zona.

2 Botas, capote, machete, mecate, binoculares y guías de campo.

1 Botas, capote, machete y mecate.

142 5. Contexto Geopolítico (CG).
A. Nacional, Binacional, Trinacional $(1,2,3)$
3
2
1

B. Límites demarcados (Presencia/ Ausencia).

1

0

6. Producción de Bienes y Servicios (PBS).

A. Presencia de instalaciones turísticas (Restaurantes, alojamiento y/o algún tipo de actividad recreativa) (A, M, B, N):

\begin{tabular}{|c|c|c|c|}
\hline \multirow{2}{*}{ Categorías } & \multicolumn{3}{|c|}{ Tamaño de Áreas Protegidas } \\
\cline { 2 - 4 } & Pequeñas & Medianas & Grandes \\
\hline 3 & Más de 3 & Más de 6 & Más de12 \\
\hline 2 & $2-3$ & $4-6$ & $8-12$ \\
\hline 1 & $0-1$ & $2-3$ & $4-7$ \\
\hline 0 & 0 & 0 & 0 \\
\hline
\end{tabular}

\begin{tabular}{|c|c|}
\hline Categoría de Tamaño & Área (Ha) \\
\hline Pequeña & Hasta 243,360 \\
\hline Mediana & $243,361-486,680$ \\
\hline Grande & $486,681-730,000$ \\
\hline
\end{tabular}


De las 76 áreas protegidas del Sistema, 13 están siendo utilizadas por el turismo nacional e internacional, predominando las actividades de turismo local. Las áreas que reciben turismo son (MARENA, 1999):

- En el Pacífico de Nicaragua:

- Parque Nacional Volcán Masaya.

- $\quad$ Laguna de Apoyo.

- Laguna de Xiloá.

- Isla de Ometepe (Volcán Maderas y Volcán Concepción),

- Chocoyero-El Brujo.

- Volcán Mombacho.

- Estero Padre Ramos.

- Isla Juan Venado.

- Chacocente.

- La Flor.

- $\quad$ En el Sureste de Nicaragua:

SI-A-PAZ, principalmente el Archipiélago de Solentiname, Los Guatuzos, la Fortaleza la Inmaculada (El Castillo) y la Gran Reserva Indio Maíz.

\section{Directrices que regulan el turismo en las áreas protegidas:}

- Limitar el acceso al público en general, salvo a personas acreditadas por autoridad competente para la realización de acciones permitidas conforme al plan de manejo del Área.

- Permitir únicamente el establecimiento y desarrollo de infraestructura y servicios con fines de investigación, vigilancia, ecoturismo, recreación y educación, en las zonas destinadas para tal fin en los planes de manejo.

- Permitir las actividades de investigación, educación, e interpretación ambiental e histórico cultural, turismo y recreación conforme a las normativas pertinentes.

Las categorías de manejo que no permiten actividades de turismo dentro de las áreas protegidas son:

1. Reserva Biológica.

2. Reserva de Recursos Genéticos.

Las categorías de manejo que permiten actividades de turismo dentro de las áreas protegidas y conforme al plan de manejo del área son:

1. Parque Nacional.

2. Monumento Nacional.

3. Monumento Histórico.

4. Refugio de Vida Silvestre.

5. Reserva Natural.

6. Paisaje Terrestre y/o Marino Protegido.

En el caso de la Reserva de Biosfera, el manejo está orientado a un sistema de zonificación que da cabida a diversas intensidades de intervención, entre ellas el turismo, respetando las disposiciones propias de las categorías de Áreas Protegidas que la integran. Para realizar las valoraciones en este punto se utilizará una tabla de contingencia como la siguiente: 


\begin{tabular}{|l|c|l|}
\hline Reglamentación & $\begin{array}{l}\text { Presencia de } \\
\text { Turismo }\end{array}$ & $\begin{array}{l}\text { Ausencia de } \\
\text { Turismo }\end{array}$ \\
\hline Se puede & $(1)$ & $\begin{array}{l}\text { Incentivar conforme al } \\
\text { plan de manejo del área }\end{array}$ \\
\hline No se puede & $(2)$ & OK \\
\hline
\end{tabular}

(1) En dependencia de la cantidad, evaluar la capacidad de carga.

(2) Implementación de sanciones por parte de la DGAP.

B. Calidad de las instalaciones turísticas (A, M, B, N):

3 Alojamiento y alimentación de calidad alta.

2 Alojamiento de calidad media y alimentación de calidad media o alguno de los dos de calidad alta y el otro de calidad media.

1 Alojamiento y alimentación de calidad baja.

0 No hay

144 C. Aporte de agua potable (Presencia/ Ausencia).

1

0

D. Cultivo de especies (Presencia/ Ausencia).

1

0

Criterios ecosistémicos: De conformidad con los objetivos y metas de la Conferencia de las Partes de la Convención de Diversidad orientados hacia el año 2010 (UNEP/CDB/COP, 2004), que enfatiza el interés en el enfoque de ecosistemas, se dará un lugar especial al monitoreo de los mismos.

La idea consiste en hacer una selección cruzada que parta de tomar como punto de referencia las áreas previamente seleccionadas mediante la matriz multicriterio, y en éstas definir cuáles ecosistemas serán priorizados para su monitoreo en los próximos años. Cuando las áreas previamente seleccionadas ya no contengan los ecosistemas seleccionados, se seguirá eligiendo de entre aquellas que tengan una mayor puntuación en la matriz.

Los ecosistemas seleccionados, llamados en estemarcoECOSISTEMASREPRESENTATIVOS, serán los siguientes (según Meyrat, 2001, simplificado y Pérez, 2002):

\section{TERRESTRES}

\section{BOSQUES LATIFOLIADOS:}

- Bosque seco (de llanura).

- Bosque semiseco (altitud mediana).

- Bosque nebliselva de montaña.

- Bosque siempreverde lluvioso.

- Bosque estacionalmente inundado.

- Bosque pantanoso.

- Bosque de Quercus. 


\section{BOSQUES NO LATIFOLIADOS:}

- Bosques de Pinos del Norte.

- Bosques de Pinos del Caribe.

\section{OTROS:}

- Yolillales.

- Sabanas de Jícaros.

- Sabanas.

- Sabanas inundadas.

- Litoral rocoso.

\section{COSTEROS}

- Litoral arenoso.

- Manglar.

\section{MARINOS:}

\section{AGUA DULCE:}

Lagos y lagunas.

- Pastos submarinos.

Ríos.

- Sabanas con gorgonáceos.

Las áreas incluidas de las que no existen PLANES DE MANEJO, se proponen porque contienen alguno de los ecosistemas representativos y/o porque en ellas tienen lugar desde hace tiempo monitoreo sistemático de algún o algunos taxa. Es el caso de las Lagunas de Apoyo y Xiloá, con sus monitoreos de peces y moluscos, los monitoreos del ecosistema y de aves en el Mombacho, etc. Se propone un grupo importante de áreas para el monitoreo de los Bosques Secos debido a que este ecosistema está realmente amenazado, existiendo escasos remanentes del mismo en la actualidad.

Cuando coinciden ecosistemas representativos con ecosistemas prioritarios, se les ha tratado de dar mayor peso haciendo una selección mayor en la cantidad de áreas a monitorear.

Otros criterios: Teniendo en cuenta la naturaleza participativa que deben tener estas actividades académicas, de cara a aportar elementos sólidos al proceso de toma de decisiones, como colofón de este proceso se realizó un taller de expertos con la comunidad científica. En el marco de este taller se propuso la inclusión de dos áreas al grupo de áreas prioritarias: 1. Apacunca, 2. Tepesomoto-Pataste. Las mismas fueron propuestas con base en algunos valores cualitativos difícilmente evaluables utilizando herramientas cuantitativas.

\section{Resultados}

Aspectos generales: En total se evaluaron 46 Áreas Protegidas. Dieciocho de éstas áreas fueron evaluadas de forma integral sobre la base de sus Planes de Manejo (Cuadros 4 y 5) y 27 fueron evaluadas sólo con base en criterios biológicos con ayuda del documento "Evaluación y Redefinición del Sistema de Áreas Protegidas de las Regiones Pacífico y Centro Norte de Nicaragua" (FUNDENIC) (Cuadro 6). 
Cuadro 4. Áreas protegidas evaluadas con la matriz multicriterio.

\begin{tabular}{|l|c|l|c|}
\hline \multicolumn{1}{|c|}{ Área } & Puntuación & \multicolumn{1}{c|}{ Área } & Puntuación \\
\hline E. Padre Ramos & $23 / 42$ & Wawashan & $26 / 42$ \\
\hline Bosawas & $33 / 42$ & Cerro Silva & $23 / 42$ \\
\hline Rió E-Chacocente & $18 / 42$ & Chocoyero el Brujo & $22 / 42$ \\
\hline Los Guatuzos & $28 / 42$ & Cayos Miskitos & $31 / 42$ \\
\hline Tisey & $25 / 42$ & Juan Venado & $27 / 42$ \\
\hline Datanlí & $18 / 42$ & $\begin{array}{l}\text { Fortaleza de la } \\
\text { Concepción }\end{array}$ & $32 / 42$ \\
\hline Cerro El Arenal & $12 / 42$ & Solentiname & $29 / 42$ \\
\hline Cerro Musún & $25 / 42$ & Rió San Juan & $37 / 42$ \\
\hline Cerro Apante & $14 / 42$ & $\begin{array}{l}\text { Miraflores } \\
\text { Moropotente }\end{array}$ & $24 / 42$ \\
& & & \\
\hline
\end{tabular}

Cuadro 5. Matriz multicriterio con los valores por áreas y por categorías de análisis.

\begin{tabular}{|c|c|c|c|c|c|c|c|c|c|c|c|c|c|c|c|c|c|c|c|c|c|}
\hline \multirow{2}{*}{ Àreas } & \multicolumn{21}{|c|}{ Categorías } \\
\hline & IA & IB & IC & ID & IIA & II B & IIC & IIIA & IIIB & IIIC & IIID & IIIE & IVA & IVB & V A & $\mathrm{VB}$ & VIA & VIB & VIC & VID & \\
\hline E. Padre Ramos & 1 & 0 & 1 & 3 & 1 & 1 & 2 & 4 & 0 & 0 & 1 & 0 & 1 & 3 & 1 & 1 & 1 & 1 & 0 & 1 & 23 \\
\hline Bosawás & 1 & 1 & 3 & 3 & 2 & 1 & 3 & 4 & 4 & 1 & 1 & 1 & 1 & 3 & 2 & 1 & 0 & 0 & 1 & 0 & 33 \\
\hline Rio E-Chacocente & 1 & $\mathbf{0}$ & 1 & 3 & 1 & 1 & 1 & 2 & 1 & $\mathbf{0}$ & 0 & $\mathbf{0}$ & 1 & 3 & 1 & 1 & 1 & 0 & 0 & $\mathbf{0}$ & 18 \\
\hline Los Guatuzos & 1 & $\mathbf{0}$ & 2 & 1 & 1 & 1 & 2 & 4 & 4 & 0 & 1 & 1 & 1 & 3 & 1 & 1 & 1 & 2 & $\mathbf{0}$ & 1 & 28 \\
\hline Tisey & 1 & 0 & 1 & 3 & 1 & 1 & 3 & 2 & 1 & $\mathbf{0}$ & 1 & $\mathbf{0}$ & 1 & 3 & 1 & 1 & 2 & 2 & 1 & 0 & 25 \\
\hline Datanli-EI Diablo & 1 & $\mathbf{0}$ & 0 & 2 & 1 & 1 & 1 & $\underline{2}$ & 4 & $\mathbf{0}$ & 0 & $\mathbf{0}$ & 0 & 3 & 1 & 1 & 0 & 0 & 1 & 0 & 18 \\
\hline Cerro El Arenal & 1 & $\mathbf{0}$ & 0 & 0 & 1 & 1 & 1 & 2 & $\mathbf{0}$ & 0 & 0 & $\mathbf{0}$ & 0 & 3 & 1 & 1 & 0 & 0 & 1 & 0 & 12 \\
\hline Cerro Musún & 1 & $\mathbf{0}$ & 1 & 2 & 1 & 1 & 2 & 2 & 4 & 1 & 0 & $\mathbf{0}$ & 1 & 3 & 1 & 1 & 1 & 2 & 1 & 0 & 25 \\
\hline Cerro Apante & 1 & 0 & $\mathbf{0}$ & 0 & 1 & 1 & 1 & 2 & 2 & 0 & 0 & 0 & 0 & 3 & 1 & 1 & 0 & 0 & 1 & 0 & 14 \\
\hline Rio San Juan & 1 & $\mathbf{0}$ & 2 & 3 & 1 & 1 & 3 & 4 & 4 & 1 & 1 & 1 & 1 & 3 & 2 & 1 & 3 & 3 & 1 & 1 & 37 \\
\hline Cerro Wawashan & 1 & 1 & 1 & 3 & 1 & 1 & 3 & 4 & 3 & 0 & 1 & 0 & 0 & 0 & 1 & 1 & 3 & 1 & 1 & 0 & 26 \\
\hline Cayos Mismitos & 1 & 1 & 3 & 3 & 2 & 1 & 3 & 4 & 4 & 0 & 1 & 1 & 0 & 0 & 1 & 1 & 3 & 1 & 1 & 0 & 31 \\
\hline Solentiname & 1 & 0 & 2 & 3 & 1 & 1 & 3 & 4 & 3 & 1 & 1 & 1 & 0 & 0 & 1 & 1 & 3 & 3 & 0 & 0 & 29 \\
\hline Isla Juan Venado & 1 & 0 & 2 & 3 & 1 & 1 & 3 & 3 & 2 & $\mathbf{0}$ & 1 & $\mathbf{0}$ & 2 & 0 & 1 & 1 & 3 & 2 & $\mathbf{0}$ & 1 & 27 \\
\hline $\begin{array}{l}\text { Inmaculada } \\
\text { Concepción }\end{array}$ & 1 & $\mathbf{0}$ & 3 & 3 & 1 & 1 & 3 & 2 & 3 & 1 & 1 & 1 & 1 & 2 & 1 & 1 & 3 & 2 & 1 & 1 & 32 \\
\hline Miraflor_Moropotente & 1 & 0 & 2 & 3 & 1 & 1 & 3 & 2 & 4 & $\mathbf{0}$ & $\mathbf{0}$ & $\mathbf{0}$ & 1 & 3 & 1 & 1 & $\mathbf{0}$ & $\mathbf{0}$ & 1 & $\mathbf{0}$ & 24 \\
\hline Cerro Silva & 1 & 1 & 1 & 2 & 1 & 1 & 3 & 4 & 4 & 0 & 1 & 1 & $\mathbf{0}$ & 0 & 1 & 1 & 0 & 0 & 1 & 0 & 23 \\
\hline Chocoyero-EI Brujo & 1 & 0 & 2 & 3 & 1 & 1 & 1 & 2 & 3 & $\mathbf{0}$ & 1 & 0 & 1 & 3 & 1 & 1 & 0 & 0 & 1 & 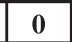 & 22 \\
\hline
\end{tabular}


Cuadro 6. Áreas protegidas que carecen de Plan de Manejo y han sido evaluadas sólo con base en criterios biológicos.

\begin{tabular}{|c|c|c|}
\hline Área Protegida & $\begin{array}{c}\text { Puntaje máximo a } \\
\text { obtener } \\
11\end{array}$ & $\begin{array}{c}\text { Puntaje } \\
\text { Base } \\
42\end{array}$ \\
\hline 21. Parque Nacional Volcán Masaya & 6 & 22 \\
\hline $\begin{array}{l}\text { 22. Parque Nacional Archipiélago de } \\
\text { Zapatera }\end{array}$ & 6 & 23 \\
\hline 23. Reserva de Recursos Genéticos Yucul & 5 & 19 \\
\hline 24. Refugio de Vida Silvestre La Flor & 4 & 15 \\
\hline 25. Reserva Natural volcán Cosigüina & 7 & 27 \\
\hline 26. Reserva Natural Delta del Estero Real & 9 & 34 \\
\hline $\begin{array}{l}\text { 27. Reserva Natural Complejo Volcánico } \\
\text { San Cristóbal }\end{array}$ & 6 & 23 \\
\hline 28. Reserva Natural Península de Chiltepe & 5 & 19 \\
\hline 29. Laguna de Asososca & 3 & 11 \\
\hline 30. Reserva Natural Laguna de Tiscapa & 2 & 8 \\
\hline 31. Reserva Natural Laguna de Tisma & 8 & 31 \\
\hline 32. Reserva Natural Volcán Mombacho & 6 & 23 \\
\hline 33. Reserva Natural Laguna de Apoyo & 7 & 27 \\
\hline $\begin{array}{l}\text { 34. Reserva Natural Cordillera de Dipilto y } \\
\text { Jalapa }\end{array}$ & 6 & 23 \\
\hline 35. Reserva Natural Tepesomoto-Pataste & 7 & 27 \\
\hline $\begin{array}{l}\text { 36. Reserva Natural Complejo Volcánico } \\
\text { Telica-Rota }\end{array}$ & 2 & 8 \\
\hline $\begin{array}{l}\text { 37. Reserva Natural Complejo Volcánico } \\
\text { Pilas-El Hoyo }\end{array}$ & 5 & 19 \\
\hline $\begin{array}{l}\text { 38. Reserva Natural Complejo Volcánico } \\
\text { Momotombo }\end{array}$ & 6 & 23 \\
\hline 39. Reserva Natural Volcán Concepción & 3 & 11 \\
\hline 40. Reserva Natural Cerro Kuskawás & 7 & 27 \\
\hline 41. Reserva Natural Laguna de Mecatepe & 6 & 23 \\
\hline 42. Reserva Natural Laguna de Nejapa & 2 & 8 \\
\hline 43. Reserva Natural Cerro Pancasán & 4 & 15 \\
\hline 44. Reserva Natural Cerro Quiabuc & 6 & 23 \\
\hline 45. Reserva Natural Sierra Quirragua & 6 & 23 \\
\hline 46. Reserva Natural Cerro Tomabú & 2 & 8 \\
\hline 47. Reserva Natural Volcán Maderas & 7 & 27 \\
\hline 48. Reserva Natural Cerro Guabule & 4 & 15 \\
\hline
\end{tabular}


Áreas Protegidas seleccionadas como prioritarias: En el Cuadro 7 yla Ilustración 5 se presenta el resumen de las Áreas Protegidas seleccionadas como prioritarias para investigación y monitoreo, así como los ecosistemas principales que serán monitoreados en ellas.

Cuadro 7. Resumen de las Áreas Protegidas seleccionadas como prioritarias.

\begin{tabular}{|c|c|c|}
\hline Área Protegida & Ecosistema (s) & Criterio \\
\hline 1. Bosawás & - Bosque Nebliselva del norte & Multicriterio \\
\hline 2. Los Guatuzos & $\begin{array}{l}\text { - Yolillales } \\
\text { - Sabanas inundadas } \\
\text { - Bosque estacionalmente } \\
\text { inundado }\end{array}$ & Multicriterio \\
\hline 3. Tisey -Estanzuela & - $\quad$ Pinares (Pinus oocarpa ) & Multicriterio \\
\hline 4. Cerro Musún & - Bosque nebliselva del norte & Multicriterio \\
\hline 5. Cayos Miskitos & $\begin{array}{l}\text { - } \quad \text { Pinares (Pinus caribaea ) } \\
\text { - Pastos submarinos de Thalassia } \\
\text { - Arreci fes de coral } \\
\text { - Sabanas de gorgonáceos } \\
\end{array}$ & Multicriterio \\
\hline 6. Juan Venado & - $\quad$ Manglares del Pacífico & Multicriterio \\
\hline 7. Cerro Silva & $\begin{array}{l}\text { - } \quad \text { Bosque siempreverde lluvioso } \\
\text { - Bosque pantanoso } \\
\text { - } \quad \text { Manglares del Caribe } \\
\end{array}$ & Multicriterio \\
\hline 8. Chacocente & $\begin{array}{ll}\text { - } & \text { Bosque seco de llanura } \\
\text { - } & \text { Litoral arenoso } \\
\text { - } & \text { Litoral rocoso } \\
\end{array}$ & Multicriterio \\
\hline 9. Chocoyero & - Bosque semiseco submontano & Multicriterio \\
\hline 10. Solentiname & - $\quad$ Lago Cocibolca & Multicriterio \\
\hline 11. Rio San Juan & - $\quad$ Ecosistema riberino & Multicriterio \\
\hline 12. Laguna de Apoyo & - Laguna continental & Criterio Biológico \\
\hline 13. Laguna de Xiloá & - Laguna continental & Criterio Biológico \\
\hline 14. Layasiksa & - Sabanas & Criterio Biológico \\
\hline 15. Mombacho & - Bosque neliselva del Pacífico & Criterio Biológico \\
\hline $\begin{array}{l}\text { 16. Complejo San Cristóbal, } \\
\text { Telica - Rota, Pilas -El Hoyo, } \\
\text { Momotombo. }\end{array}$ & 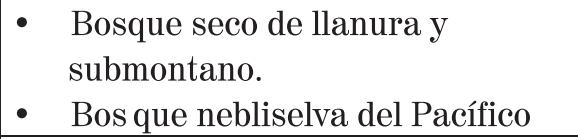 & Criterio Biológico \\
\hline 17. Apacunca & $\begin{array}{l}\text { - Población de Zea nicaraguensis } \\
\text { (Maíz endémico nacional) }\end{array}$ & $\begin{array}{l}\text { Otros (Propuesta en } \\
\text { el taller) }\end{array}$ \\
\hline 18. Tepesomoto -Pataste & $\begin{array}{l}\text { Bosque de Quercus (Roble } \\
\text { encino) }\end{array}$ & $\begin{array}{l}\text { Otros (Propuesta en } \\
\text { el taller) }\end{array}$ \\
\hline
\end{tabular}




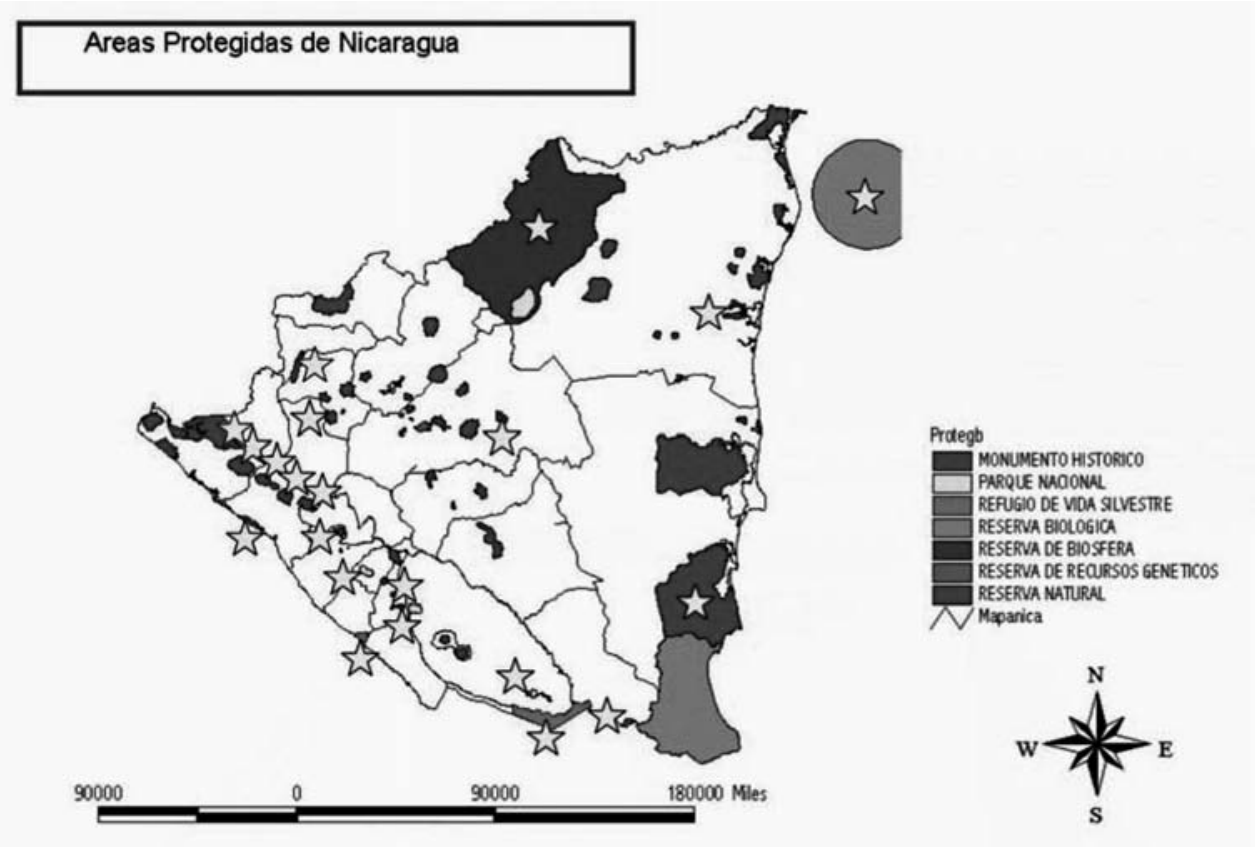

Ilustración 5. Mapeo de las áreas prioritarias en el país.

\section{Conclusiones}

1. En el marco de la elaboración del programa de investigación y monitoreo de AP de Nicaragua, se diseñó una herramienta para la valoración estructural general de las áreas protegidas del país.

2. Como resultado de la aplicación de la citada herramienta, se construyó un portafolio de 16 áreas protegidas prioritarias para el país.

3. Como complemento a la aplicación de la herramienta se realizó un taller de expertos, en cuyo marco se propusieron dos nuevas áreas sobre la base de algunos valores peculiares difíciles de detectar por herramientas cuantitativas, lo cual hace un total de 18 áreas protegidas prioritarias para el país.

\section{Agradecimientos}

Agradecemos al Maestro Germán Cruz, del Proyecto ARAUCARIA-MARENA, sus importantes comentarios que contribuyeron a la mejoría de este trabajo, así como su sugerencia en relación con la evaluación de las áreas protegidas que dio origen al diseño de la herramienta multicriterio presentada en este artículo. También a la Lic. Liliana Díaz, de la DGAP-MARENA.

A los colegas de la comunidad científica del país por sus comentarios invaluables, en especial a los Drs. Ricardo Rueda, del Herbario HULE, y Jean Michel Maes, del MEL. 


\section{Referencias bibliográficas}

-BRIGEWATER, P. (1999). "Prefacio". En:Monitoreo biológico en la selva maya. Guatemala: MAB-TED-WCS.

-CONESA, V. (1995). Guía metodológica para la evaluación del impacto ambiental. Madrid: Mundi Prensa.

-GARCÍA, R. (2002). Biología de la conservación: conceptos y prácticas. San José: PNUD/ CBM/ INBio.

Ley 217. "Ley General del Medio Ambiente y los Recursos Naturales y sus Reglamentos", Publicada en La Gaceta, Diario Oficial, No. 105, del 06 de Junio de 1996.

-MARENA (1999). Biodiversidad en Nicaragua: Un estudio de país. Managua: MARENA, PANIF. Primera edición.

-MARENA (2001). Estrategia nacional de biodiversidad. Managua: Imprimatur.

-MARTÍNEZ-SÁNCHEZ, J.; MAES, J.; VAN DEN BERGHE, E.; MORALES, S. y CATAÑEDA, E. (2001). Biodiversidad zoológica en Nicaragua. Managua: PNUD, GEF, MARENA.

-MENA, Y. y ARTAVIA, G. (s.f.).Hacia la administración eficiente de las áreas protegidas:

Políticas e indicadores para su monitoreo. Costa Rica: Editorial INBio.

-MEYRAT, A. (2001). "Estado de conservación de los ecosistemas de Nicaragua". En: Estrategia Nacional de Biodiversidad. Managua: Imprimatur.

-McGUINNES, B. (1994). A heritage for the 21st century: conserving Pennsylvania s native biological diversity. Pennsylvania: A report by the Pennsylvania technical committee.

-McNEELY, J. (2002). The role of taxonomy in conserving biodiversity: an UICN perspective. Pretoria: Bionet Global Taxonomy Workshop.

-MILÁN, J. (2004). Manual de Estudios Ambientales para la Planificación y los Proyectos de Desarrollo. Managua: UNI, Primera edición.

-MITTERMEIER, R.; MYERS, N. y MITTERMEIER, C. (2000). Hotspots: Earth's biologically richest and most endangered terrestrial ecoregions. Conservation International.

-PÉREZ, A. (2002). Propuesta de Sistema Nacional de Monitoreo de la Biodiversidad. Informe final. Managua: MARENA/PNUD/GEF.

-RALLO, A. (1998). Principios y método en sistemática y taxonomía. Informe. España: Universidad del País Vasco.

-UICN (1994). Categorías de las listas rojas. Suiza: Gland.

-UNEP/CDB/COP (2004). Integración de las metas orientadas a la obtención de resultados ...por la cumbre mundial sobre el desarrollo sostenible. Addendum Kuala Lumpur: UNEP/CDB/COP.

-VOZMEDIANO, J. (2003). Hacia una consolidación jurídica y social del Programa MAB (Man and Biosphere). Madrid: FAES. 